\title{
Journal of Global Optimization Best Paper Award for a paper published in 2014
}

\author{
Sergiy Butenko 1
}

Received: 30 October 2015 / Accepted: 5 November 2015 / Published online: 14 November 2015

C) Springer Science+Business Media New York 2015

The fourth edition of the annual Journal of Global Optimization (JOGO) Best Paper Award, which consists of $\$ 1000$ and a certificate, considered papers published in 2014 issues of JOGO. The award committee that included Mirjam Dür, Simge Kucukyavuz, Panos Pardalos, and Anatoly Zhigljavsky, faced a challenging task of selecting the best of nine outstanding papers that were nominated. Two rounds of voting yielded three clear favorites with nearly identical level of support by the committee. Thus, the award will be split between the three papers, listed below in the order of their appearance in JOGO.

$\diamond$ Immanuel M. Bomze, Stefan Gollowitzer, and E. Alper Yildirim, Rounding on the standard simplex: regular grids for global optimization. (volume 59, pages 243-258).

\begin{abstract}
Given a point on the standard simplex, we calculate a proximal point on the regular grid which is closest with respect to any norm in a large class, including all $\ell^{p}$-norms for $p \geq 1$. We show that the minimal $\ell^{p}$-distance to the regular grid on the standard simplex can exceed one, even for very fine mesh sizes in high dimensions. Furthermore, for $p=1$, the maximum minimal distance approaches the $\ell^{1}$-diameter of the standard simplex. We also put our results into perspective with respect to the literature on approximating global optimization problems over the standard simplex by means of the regular grid.
\end{abstract}

$\diamond$ Remigijus Paulavicius, Yaroslav D. Sergeyev, Dmitri E. Kvasov, and Julius Žilinskas, Globally-biased DISIMPL algorithm for expensive global optimization (volume 59, pages 545-567).

Abstract. DIRECT-type global optimization algorithms often spend an excessive number of function evaluations on problems with many local optima exploring suboptimal local minima, thereby delaying discovery of the global minimum. In this paper, a globally-biased simplicial partition DISIMPL algorithm for global optimization of

\section{Sergiy Butenko}

butenko@tamu.edu

1 Texas A\&M University, College Station, TX, USA 
expensive Lipschitz continuous functions with an unknown Lipschitz constant is proposed. A scheme for an adaptive balancing of local and global information during the search is introduced, implemented, experimentally investigated, and compared with the well-known DIRECT and DIRECTl methods. Extensive numerical experiments executed on 800 multidimensional multiextremal test functions show a promising performance of the new acceleration technique with respect to competitors.

$\diamond$ Angelos Tsoukalas and Alexander Mitsos, Multivariate McCormick relaxations (volume 59, pages 633-662).

\begin{abstract}
McCormick (Math Prog 10(1):147-175, 1976) provides the framework for convex/concave relaxations of factorable functions, via rules for the product of functions and compositions of the form $F \circ f$, where $F$ is a univariate function. Herein, the composition theorem is generalized to allow multivariate outer functions $F$, and theory for the propagation of subgradients is presented. The generalization interprets the McCormick relaxation approach as a decomposition method for the auxiliary variable method. In addition to extending the framework, the new result provides a tool for the proof of relaxations of specific functions. Moreover, a direct consequence is an improved relaxation for the product of two functions, at least as tight as McCormicks result, and often tighter. The result also allows the direct relaxation of multilinear products of functions. Furthermore, the composition result is applied to obtain improved convex underestimators for the minimum/maximum and the division of two functions for which current relaxations are often weak. These cases can be extended to allow composition of a variety of functions for which relaxations have been proposed.
\end{abstract}

Congratulations to the authors of the awarded papers! I would also like to thank Springer for sponsoring this award and the committee members for their active involvement. Nominations for the 2015 JOGO Best Paper Award should be emailed to butenko@tamu.edu. All JOGO papers published in 2015 (volumes 61-63) are eligible. 